\title{
BUDDHISMUS UND STAAT IN SÜDOSTASIEN
}

In vier Staaten Hinterindiens, nämlich in Burma (Birma), Thailand (Siam), Kambodscha und Laos, ist noch heute die buddhistische Religion der wichtigste Faktor für die Integrierung der Bevölkerung jener Gebiete. Die politischen, wirtschaftlichen, militärischen und sozialen Verhältnisse dieser vier südostasiatischen Staaten werden vorwiegend vom Buddhismus her bestimmt und sind nur von dort her zureichend und über die historischen Einzelgeschehnisse hinausgehend zu interpretieren. Für eine solche Betrachtung ist es notwendig, zunächst einmal auf die Wurzeln dessen zurückzugehen, was man eine buddhistische Staatstheorie nennen kann. Sodann wäre zu zeigen, wie sich diese Idee in den vom Buddhismus beeinflußten oder beherrschten Staaten Asiens ausgeprägt hat, wobei die Unterschiede zwischen den vier südostasiatischen Staaten sowie Ceylon (das eine Sonderstellung einnimmt) einerseits und den Ländern Ostasiens (einschließlich Vietnam) andererseits wenigstens kurz gestreift werden müssen. Auf dieser Grundlage können dann für Südostasien einige Betrachtungen über das Problem angestellt werden, das wir das Verhältnis von Kirche und Staat nennen. Bei dieser Untersuchung ist es als günstig zu verzeichnen, daß jüngst in Deutschland ein Werk erschienen ist, welches in reicher Fülle Material zur Lage des modernen Staates in den buddhistischen Gebieten zur Verfügung stell $\mathrm{t}^{2}$. Auf dieses Werk des Göttinger Indologen Professor Bechert wird daher im Laufe der Darstellung, besonders im 2. Teil, immer wieder hinzuweisen sein.

I.

Um das Verständnis für die Situation der buddhistischen Staatsauffassung zu erleichtern, erscheint es als dienlich, diese zunächst mit den Verhältnissen von Staat und Religion zu vergleichen, die beim europäischen Denken über diese Frage im Vordergrund stehen.

Die drei Weltreligionen, die nacheinander aus dem vorderasiatisch-semitischen Raum hervorgegangen sind, nämlich Judentum, Christentum und Islam, müßten sowohl unter ihren Gründern als auch unter ihren Nachfolgern immer wieder mit staatlichen Mächten kämpfen. Moses mußte gegenüber der Feindschaft der Ägypter, Mohammed gegenüber der Feindschaft der Mekkaner, Christus gegenüber der Feindschaft der Pharisäer die neue Lehre behaupten und mit mehr oder weniger Schwierigkeiten durchsetzen. Und auch später hatten die drei Religionen unaufhörliche Auseinandersetzungen zu bestehen: Die Juden mußten in Palästina ihre Religion gegen andere Völker durchsetzen; der Islam stand unter seinen Kalifen in permanentem Kriegszustand mit den Staaten der Ungläubigen, und das Christentum war, nachdem es 300 Jahre lang in Kampfstellung zum römischen Reich gestanden hatte, als Staatsreligion des abendländischen Kaisertums in unaufhörlich wogendem Ringen um die Frage, welchem der zwei Reiche die Vorherrschaft gebühre, dem weltlichen Kaisertum oder dem geistlichen Regiment des Papstes. Aus dieser historischen Situation erklärt es sich, daß die modernen Staaten

\footnotetext{
1 Die englische Schreibweise (Burma) sei hier der von der französischen (Birmanie) abgeleiteten (Birma) wegen ihrer internationalen Verbreitung vorgezogen. Die genaue Umschrift im Deutschen wäre Barma.

- Heinz Bechert: Buddhismus, Staat und Gesellschaft in den Ländern des Theravāda-Buddhismus (Schriften des Instituts für Asienkunde in Hamburg, Bd. XVII/1-3).

Bd. I: Allgemeines und Ceylon, Frankfurt a. M. 1966, 375 S.

Bd. II: Birma, Kambodscha, Laos, Thailand, Wiesbaden 1967, 377 S.

Bd. III: in Vorbereitung.
} 
des christlichen Abendlandes, des islamischen Orients und des neuen Israel in zunehmendem Maße eine Trennung von Kirche und Staat befürworten und praktizieren. Dem Streben des modernen Staates nach allseitiger und absoluter Zuständigkeit zur Regelung der irdischen Lebensverhältnisse stand der Machtanspruch der Religionen konkurrierend gegenüber. In diesem Machtkampf siegte der Staat, indem er die Religion auf das geistliche Gebiet beschränkte, dessen Gewichtigkeit aber bei den Massen immer mehr abnahm. Die Trennung von Kirche und Staat bedeutet daher: Alles Wesentliche regelt der Staat nach seinem Ermessen, die Kirche darf nur noch dort tätig sein, wo es dem Staat unwichtig erscheint oder sie darf als Auftragsverwaltung in seinen Diensten stehen.

Dieser notgedrungen kurzen und darum etwas pointiert klingenden Skizze bedarf es, um demgegenüber die sehr andere Situation des Buddhismus abzuheben. Nur aus dieser Gegenüberstellung ergibt sich sodann die Möglichkeit zu entscheiden, ob überhaupt vergleichbare Verhältnisse vorliegen.

Die buddhistische Religion unterscheidet sich schon durch ihre Begründung wesentlich von den drei anderen Weltreligionen, nämlich insofern, als hier keinerlei Kampf mit weltlichen Mächten vorlag. Der Prinz Siddhattha Gotama aus dem Himālaya-Fürstentum der Sakyer begann in seinem 35. Lebensjahr seine Lehre als Buddha (= Erwachter) zu verkünden und lehrte als Wandermönch 45 Jahre lang in Mittelindien. Er gründete als erstes einen Orden (Sangha = Gemeinschaft), in welchem sich seine Jünger zu einem Leben der Selbsterziehung zusammenfanden und in dessen Verfassung autoritäre und demokratische Elemente wohl abgewogen sind. Dieser älteste Orden der Welt hatte keinerlei Absichten, sich in weltliche Verhältnisse einzumischen, ja dies widersprach sogar seinen rein geistlichen und meditativen $Z$ wecken; auch eine Mission war darin nicht wesentlich, sondern ergab sich mehr indirekt durch die indische Gewohnheit, bei Angehörigen solcher Religionsgemeinschaften um Rat zu fragen. Der Orden wurde ausschließlich von Laien unterhalten, d.h. von solchen, die das strenge Mönchsleben noch nicht führen konnten, aber sich zur Lehre des Gründers bekannten und sich auf dem Gebiete der Wohltätigkeit darin übten. $\mathrm{Zu}$ diesen Laien gehörten auch die Großkönige (Mahārājā) der beiden Hauptreiche des Gangesgebietes, nämlich König Pasenadi von Kosalo und Kasi (Benares) sowie König Seniyo Bimbisāro von Magadha. Sie ließen sich vom Buddha in vielen Gesprächen belehren und ermöglichten durch ihre Haltung die Ausbreitung des Buddhismus in ihren Reichen. Es war hier nicht so, daß durch die Bekehrung der Staatsoberhäupter damit an sich auch schon die Untertanen mit gewonnen wurden. Der Grundsatz „Cuius regio, eius religio" war hier unbekannt. Vielmehr entwickelte sich der Buddhismus allein durch seine Überzeugungskraft und durch das Vorbild seiner Anhänger, seien es Mönche oder Laien. Da die beiden Könige bemüht waren, die sittlichen Grundsätze des Buddhismus zu praktizieren, strahlten sie von dort her auch wieder auf die Bevölkerung aus, wobei es für die Echtheit der Überlieferung spricht, daß beide Herrscher auch in ihren Unvollkommenheiten geschildert werden. Während ihrer über dreißigjährigen Regierungszeit herrschte Friede zwischen den beiden Reichen, ein auch damals seltener Zustand. Das Verhältnis zwischen der staatlichen Macht und dem Orden war damals wie folgt zu charakterisieren: Durch das Vorbild des Religionsgründers wurden die Mönche beeinflußt, dem nachzueifern; durch das Vorbild des Ordens wurden die Könige entsprechend beeinflußt und durch das Vorbild der Könige und des Hofes wurde das ganze Land sittlich-geistig gehoben. Dazu bedurfte es keiner Macht und keines Zwanges, sondern die Sache wirkte durch sich. 
Diese Situation des Ur-Buddhismus blieb seither für die buddhistischen Staaten als Ideal bestimmend. Im Gegensatz etwa zu Platons Idealstaat und anderen Utopien hatte das buddhistische Modell den Vorzug, im Verlaufe seiner Geschichte immer wieder verwirklicht zu werden. Der buddhistische Kaiser Asoka von Magadha, der Indien einigte (273-236 v. Chr.), ist zwar das berühmteste, aber durchaus nicht das einzige Beispiel dessen, was in den buddhistischen Texten ein Gesetzeskönig (dhamma-rājā) bzw. Universalmonarch (Cakkavatti) genannt wird. In Indien waren die Kaiser Kanishka (c. 10-80 n. Chr.) und Harshavardhana (606-648) die ruhmreichsten Vorbilder solcher Herrscher. Von letzterem heißt es:

„Eine gute Verwaltung, humane Rechtspflege, niedrige Steuern, Krankenhäuser für Mensch und Tier, Blüte der Künste, Begeisterung für die Verbreitung des Glaubens, Abschaffung des Fleischgenusses und dergleichen werden als Kennzeichen und Errungenschaften seiner Regierung gerühmt. “3

A uch in den buddhistischen Nachfolgestaaten innerhalb und außerhalb des indischen Kontinentes finden sich im Verlaufe der wechselvollen Geschichte immer wieder solche Herrscher, wie unter der Pāla-Dynastie in Bengalen (730-1197), wie in allen vier südostasiatischen Staaten und wie besonders unter den c y lon es i$\mathrm{s} \mathrm{ch}$ e $\mathrm{n}$ Königen, die unter Asoka zum Buddhismus bekehrt wurden und bis zu ihrem Sturze durch die Engländer (1815) das buddhistische Königtum meist recht würdig repräsentierten. Trotzdem besteht zwischen Ceylon und den unten näher $\mathrm{zu}$ erörternden hinterindischen Staaten ein nicht unerheblicher Unterschied, der auch für die heutige Lage von Bedeutung ist. Dieser Unterschied liegt darin, daß Ceylon am längsten unter Kolonialherrschaft gelebt hatte: Thailand war nie Kolonie, Burma nur 60 Jahre lang und Kambodscha und Laos hatten als französische Protektorate ihre interne Eigenständigkeit ungebrochen bis in die Gegenwart bewahrt. Ceylon hingegen war, jedenfalls im Küstenland, zuerst portugiesisch, dann holländisch und schließlich englisch: diese Herrschaftsformen haben ihre Spuren hinterlassen und haben dazu geführt, daß in Ceylon westliche Einflüsse stärker waren als anderswo. Hier kam es zu einer weitgehenden Durchdringung buddhistischer und abendländischer Gedanken, was auch dadurch begünstigt wurde, daß Ceylon als vorwiegend indo-arisches Land rassisch dem Europäer nähersteht als die mongolischen Rassen Hinterindiens. In Bezug auf den Staat ist es so, daß Ceylon das einzige der genannten buddhistischen Länder ist, in dem eine parlamentarische Demokratie (und zwar heute als konstitutionelle Monarchie innerhalb des Commonwealth) schon seit der Zeit vor der Unabhängigkeit wirklich funktioniert hat. Andererseits ist Ceylon derjenige Staat, in welchem der Sangha entgegen seiner andersartigen Tradition am stärksten politisiert. In den anderen Staaten traten politisierende Mönche hauptsächlich in der Zeit des Unabhängigkeitskampfes hervor, da der Sangha die stärkste Institution des nationalen Selbstbewußtseins war. Nach Erreichung der Unabhängigkeit von Burma, Kambodscha und Laos verlor diese Betätigung ihren Sinn und man kehrte weitgehend wieder zu der traditionellen Haltung rein geistiger Beeinflussung des Staates zurück. In Ceylon war es umgekehrt: vor der Unabhängigkeit kam es kaum $\mathrm{zu}$ buddhistisch bestimmten Agitationen ${ }^{4}$, um so mehr aber nach der Unabhängigkeit unter den Bandanaraikes, bis diese Phase 1965 wieder einer ruhigeren Entwicklung Platz machte. Wegen der starken Einmischung des ceylonesischen Sangha in die politischen Verhältnisse ist es verständlich, daß für Ceylon noch am ehesten die Forderung moderner Staaten paßt, die einer Macht der „Kirche“ ablehnend gegenüberstehen.

s Günther Schulemann, Geschichte der Dalai-Lamas, Leipzig 1958, S. 48.

- Bechert I, S. 83 und 307. 
Die Verhältnisse in Südostasien sind aber noch in einer zweiten Hinsicht von denen anderer buddhistischer Gebiete abzugrenzen, nämlich von dem großen Bereich des sogenannten nördlichen Buddhismus, auch Mahāyāna (Großes Fahrzeug) im Gegensatz zum südlichen Buddhismus des kleinen Fahrzeuges (Hinayāna), heute allgemein bezeichnet als Theravāda (= Lehre der Älteren). Mit diesen beiden buddhistischen Konfessionen hat es folgende Bewandtnis:

Etwa um die Zeitwende spaltete sich der Buddhismus in Indien in diese beiden Richtungen, die aber bis ins zehnte Jahrhundert dort friedlich nebeneinander lebten, ohne daß es jemals zu solchen Gegensätzen wie im Abendlande gekommen wäre. Als der Buddhismus in Indien im zehnten Jahrhundert verfiel und die mohammedanische Eroberung und die brahmanische Renaissance das Land beherrschten, kam es zu einer räumlichen Trennung in die nördliche und südliche Schule. Im Norden Indiens waren Tibet, China, Korea und Japan sowie davon ausstrahlend die Mongolei und im Süden Vietnam (Annam) vom MahāyānaBuddhismus erreicht und weitgehend geprägt worden. In den südlichen und südöstlichen Randgebieten Indiens blieb der Theravāda-Buddhismus bis heute herrschend. Ein wesentlicher Unterschied beider Richtungen besteht darin, daß der Theravāda-Buddhismus von einem festen Kern (dem Pāli-Kanon) ausgeht, in welchem die Lehre und die Organisation des Ordens geregelt sind, so daß für individuelle Eigenheiten wenig Gelegenheit ist. Die alten Sekten dieser Richtung sind daher lange ausgestorben und es gibt in dieser Konfession heute nur einen einheitlichen Buddhismus. Im Māhāyāna hingegen ist das alltägliche sowie das meditative Leben viel individueller und weit persönlicher auf den jeweiligen Lehrer oder Abt zugeschnitten: hier findet sich eine Fülle von Sekten, ohne daß in den vielen Lehrtexten ein dem Pāli-Kanon vergleichbarer einheitlicher Kern vorhanden ist. In der Geschichte hat sich nun gezeigt, daß dieser Individualismus, der jeden nach seiner Façon selig werden ließ, nur fruchtbar war, solange die Menschen ein hohes Maß an Selbstdisziplin bereits mitbrachten. Wo dies schwand, da verfiel auch der Mahāyāna-Buddhismus. Daher ist es nicht ohne Grund, daß der Kommunismus heute ausschließlich in Ländern des Mahāyāna-Buddhismus Fuß fassen konnte, nämlich in China, Tibet, der Mongolei, in Vietnam und (Nord-)Korea. Dabei ist mit zu berücksichtigen, daß der nördliche Buddhismus außer in Tibet (und der von dort bekehrten Mongolei) nie die einzige Religion, nie Staatsreligion war. Wenn auch manche chinesischen und japanischen Kaiser Buddhisten waren, so blieb der Buddhismus doch neben den chinesischen Systemen des Konfuzianus und Taoismus sowie dem japanischen Shintoismus eine Religion neben anderen. Auf den Staat aber wirkten auf die Dauer die alten Religionen stärker ein, zumal der chinesische Kaiser als „Sohn des Himmels" bzw. der japanische Tenno als "Abkömmling der Sonnengöttin“ den Staat in seiner Vergöttlichung bestimmten. Eine entsprechende Vergöttlichung im Sinne einer buddhistischen Theokratie gab es nur in Tibet, das wegen seiner einzigartigen Sonderstellung im Rahmen dieser Betrachtung außen vor bleiben kann: In Tibet sind am ehesten Vergleiche mit der abendländischen Kirche und dem Papsttum möglich. Insoweit fällt Tibet aber gerade aus dem buddhistischen Rahmen heraus ${ }^{5}$.

Im Zusammenhang mit dem Mahāyāna-Buddhismus muß auch die Lage in $\mathrm{V}$ i e t $\mathrm{n}$ a $\mathrm{m}$ gesehen werden. Das Land Annam war nicht weniger als $1000 \mathrm{Jahre}$ lang chinesisch gewesen (111 v. Chr. bis 939 n. Chr.), und diese Herrschaft hat ihm bis heute seinen Stempel aufgeprägt. Die Staatstheorie war dort im wesentlichen

\footnotetext{
5 Siehe dazu die ausführliche Besprechung des in Anmerkung 3 genannten Werkes auf S. 225 ff dieses Heftes.
} 
konfuzianisch und die Herrscher bildeten sich nach dem Vorbild der chinesischen Kaiser. Der Buddhismus von Vietnam war ebenfalls ein Spiegelbild des chinesischen Mahāyāna. So wie in China 12 und in Japan 16 Schulen individueller Art hervorgetreten waren, so bildeten sich auch in Vietnam 16 "Sekten“ heraus ${ }^{6}$. Neben ihnen bestehen gewisse synkretistische Richtungen, die im 20. Jahrhundert neue Religionen gründeten, insbesondere die Cao-Dai, Hoa-Hao und Binh-Xuyen. Nachdem der Buddhismus in Vietnam unter französischer Herrschaft eine Verfallsperiode durchgemacht hatte, kam es seit 1920 wieder zu einer Neubelebung7, in deren Linie auch die bekannten Ereignisse der „Buddhistenkrise“ Südvietnams von 1963 liegen. Theravāda-Buddhisten gibt es in Vietnam nur wenige: beide Richtungen leben aber wohlwollend nebeneinander ${ }^{8}$. Die besonderen Verhältnisse des zum Synkretismus neigenden Vietnam sind von Bechert in einem Exkurs ausgiebig dargestellt worden, wobei dem kaum etwas hinzuzufügen sein dürfte. Bechert behandelt Vietnam in seinem Buche über den Theravāda-Buddhismus hauptsächlich deshalb, weil die Verhältnisse in Kambodscha und Laos ohne eine Erörterung derjenigen in Vietnam nicht so gut verständlich sind und weil aus der Kontrastwirkung gerade die Eigenheiten der anderen buddhistischen Staaten deutlicher hervortreten.

II.

Wenn nun speziell die Situation der vier hinterindischen Staaten betrachtet wird, so lassen sich, über die nicht zu leugnenden Unterschiede hinaus, gewisse Gemeinsamkeiten feststellen: die das buddhistische Staatsideal näher konkretisieren. Davon soll im folgenden die Rede sein:

1. Alle vier Staaten besaßen eine Institution, die außer in Burma noch heute besteht, nämlich eine nationale Ordensspitze, den Sangha-rājā. Der Name, welcher Ordenskönig bedeutet, könnte dazu verführen, an eine geistliche Machtposition im Sinne eines National-Papstes zu denken. Das ist jedoch nicht der Fall: Der Sangharājā des jeweiligen Landes ist weder eine politische noch eine geistliche Machtposition, sondern nur Verwaltungsspitze, eine Art oberster Kirchenpräsident oder Patriarch. Seine Aufgabe besteht darin, durch sein Vorbild in zwei Richtungen zu wirken: einmal für die Disziplin innerhalb des Ordens und zum anderen als Bewahrer der Lehrtradition. In diesen beiden Funktionen tritt er auch dem Staate gegenüber auf und stimmt die Probleme mit diesem ab, die sich in der Praxis ergeben, wobei technische Fragen im Vordergrund stehen. Daß in Siam der Bruder des Königs Chulalongkorn im vorigen Jahrhundert lange dieses Amt innehatte, wirkte sich sehr harmonisierend aus und wird als Symbol für die brüderliche Haltung zwischen Staat und Orden angesehen. Daß in Ceylon seit dem 18. Jahrhundert kein Sangha-rājā mehr bestellt wurde ${ }^{9}$ und daß dieses Amt in Burma nach Unterbrechungen und Beschränkungen 1938 endgültig endete ${ }^{10}$, hat sich nicht gerade günstig für die Disziplin und das sittliche Niveau des Ordens ausgewirkt. Die Errichtung einer kollegialen Zentrale der Religion in Burma (Union of Burma Sāsana Council) erwies sich als kein Ersatz und dauerte nur kurze Zeit (1950 bis 1962). Wenn auch der jeweilige Sangha-rājā nicht etwa „Stellvertreter Buddhas auf Erden" war, so repräsentierte er doch auch als primus inter pares im Orden eine sittliche Autorität, die integrierende Kraft besaß.

6 Bechert II, S. 328

7 Bechert II, S. 325.

8 Bechert II, S. 329/330.

9 Bechert I, S. 211.

10 Bechert II, S. 20/21. 
2. Die enge Verbundenheit zwischen Staat und Orden zeigt sich auch darin, daß in jenen Ländern sehr viele Männer in ihrer Jugend einige Zeit im Kloster als Mönch verbringen und so für ihr ganzes Leben mit den religiösen Werten vertraut werden. Die Gemeinschaftserziehung, die in anderen Staaten dem Militär zugeschrieben wird, liegt hier im Kloster. Dabei stimmt unsere abendländische Vorstellung vom Kloster, die vorwiegend von der Regel des heiligen Benedikt bestimmt wird, keineswegs mit dem buddhistischen Kloster überein, das weit mehr Beziehung zum Leben außerhalb hat, ohne daß aber gerade eine Absicht der Einmischung bestand. Man kann vielleicht sagen, daß die Mauer des buddhistischen Klosters im Geiste besteht, nicht aber in einer äußeren Abgeschlossenheit. Im Hinblick auf die Klosterschulen für Kinder-Unterricht mögen sich Buddhismus und Christentum ähneln, aber die Funktion des Klosters als eine Art „Ordensburg“ zur Vorbereitung des jungen Mannes auf sein Laienleben im Staate ist spezifisch buddhistisch. In Thailand erhalten Beamte und Offiziere noch heute bezahlten Urlaub, wenn sie einen mehrmonatigen Klosteraufenthalt absolvieren ${ }^{11}$. So verbrachten z. B. U Nu und König Sihanouk von Kambodscha einige Zeit als Mönch im Kloster. Diese buddhistische Tradition färbte dann auch auf christliche Klöster in Hinterindien $a b$, so verbrachte z. B. Präsident Diem von Südvietnam vor dem Kriege längere Zeit in einem dortigen katholischen Kloster ${ }^{12}$.

3. Andererseits war der Orden aber auch eine Art Asyl für Politiker, die in der Welt Schiffbruch erlitten hatten. So lebt z. B. U Nu heute als Mönch in Rangun, ebenso wie einer der Hauptmitarbeiter General $\mathrm{Ne}$ Wins, der stellvertretende Generalstabschef Brigadier Aung Gyi, der sich gegen die Perpetuierung der Militärdiktatur ausgesprochen hatte ${ }^{13}$. Und in Siam verbrachte der spätere König Mongkut sogar 27 Jahre als Mönch, nachdem sein Bruder ihm die Königswürde streitig gemacht hatte. Das Kloster ist also in dieser Hinsicht eine Stätte, um eine humane Lösung weltlicher Konflikte zu ermöglichen und den gescheiterten Staatsmännern eine Existenz zu ermöglichen, die ihren Gegnern jede Furcht nimmt. Denn der Mönch hat sich nicht um Politik zu kümmern und Verstöße geen diesen Grundsatz haben stets dem Sangha und dem Staat geschadet.

4. Der buddhistischen Grundhaltung, wonach vom Charakter des Individuums die äußeren Verhältnisse, insbesondere das Wohl des Staates abhängt, entspricht es, daß die buddhistischen Herrscher dem Ideal um so näher kamen, als sie selber als Vorbild erzieherisch wirkten. Nicht Gewalt und Zwang sind dann die hauptsächlichen Formen staatlicher Betätigung, sondern Appell an sittliche Eigenschaften. So sagt Bechert: „Der Gesamthaltung U Nus entsprach es, Gesetze nicht durch strenge Maßnahmen, sondern durch ethische Ermahnungen zur Geltung bringen zu wollen." ${ }^{14}$ Dabei ging U Nu davon aus, daß seine verdienstvollen Werke als Staatschef einerseits seiner eigenen religiösen Erziehung zugute kommen würden, andererseits aber den Burmesen ein buddhistisches Leben mit dem Streben zur Erlösung erleichterten. Im Zusammenhang damit trat $\mathrm{U} \mathrm{Nu}$ immer wieder als Mahner auf, der die Bevölkerung zu einer der Religion entsprechenden Lebensführung aufrief. So sagt er:

"If we go to the root causes of the present disorder in this country, we will find that not less then $80 \%$ of them are due to apathy to religion." 15

13 Bechert II, S. 160.

14 Bechert II, S. 170.

15 Bechert II, S. 169. 
Hier mischte sich also nicht die Kirche in Belange des Staates ein, und der Staat bemühte sich nicht, den Einfluß der Kirche zurückzudrängen, sondern der Staatschef selber trat als "Animator" für die Religion im Volke auf und holte sich vom Orden den Rat weiser Mönche ${ }^{16}$. So griff er auf die Tradition des burmesischen Königstums zurück, das schon immer als Förderer und Schützer der Religion aufgetreten war. Wenn auch $\mathrm{U} \mathrm{Nu}$ das bekannteste Beispiel einer Anknüpfung an die buddhistischen Idealherrscher darstellt, so ist seine Haltung doch auch in den anderen Ländern als Grundsatz zu finden. Der Orden kann im Verhältnis zum Staat in gewisser Weise mit einem informellen Alltestenrat, einer faktischen zweiten Kammer, verglichen werden, die gegenüber den Fragen der Tagespolitik von höherer Warte gute Ratschläge gibt, falls der Staat es wünscht.

5. Die buddhistische Grundhaltung vom entscheidenden Wert der sittlichen Erziehung des Menschen bestimmt auch das Verhältnis zum Marxismus. Da der Materialismus das Heil von der Veränderung der äußeren Verhältnisse erwartet, der Buddhismus aber von der Änderung des Menschen selber, so betrachtet der Buddhist den Marxismus zwar nicht als absoluten Gegensatz zum Buddhismus, aber doch als noch unvollkommene Lehre im Sinne von U Nus Kriegsminister, $\mathrm{U}$ Ba Swe:

„Wenn wir die beiden Philosophien voneinander absetzen wollen, dann können wir mit Sicherheit annehmen, daß der Marxismus einer niedrigeren Ebene als der Buddhismus angehört. Die marxistische Theorie bezieht sich auf weltliche Angelegenheiten und sucht materielle Lebensbedürfnisse zu befriedigen. Die buddhistische Philosophie jedoch befaßt sich mit geistigen Dingen, mit dem Ziel ... der Befreiung. "17

Wenn z. B. U Nu nach dem Kriege eine Identität von Marxismus und Buddhismus (insbesondere in der Haltung zum Eigentum und zum Theismus) zu sehen glaubte, so bezeichnete er sie doch bald als bedauerlicher Irrtum, der auf einer ungenauen Kenntnis beider Lehren beruht habe ${ }^{18}$. Daran hat sich auch unter der Militärregierung $\mathrm{Ne}$ Win nicht viel geändert. Zwar hat diese die Politik der aktiven Förderung der Religion eingestellt, aber maßgebliche Mönchsvertreter bezeichnen den burmesischen Weg zum Sozialismus in der Revolutionsregierung als einen „mittleren Weg " (das ist eines der buddhistischen Ideale), der akzeptabel sei"19. Betrachtet man die Parteiphilosophie Ne Wins mit ihren buddhistischen Ausführungen, so dürfte man Bechert zustimmen, wenn er sagt, dies sei „nichts anderes als ein säkularisierter buddhistischer Sozialismus, keine echte Abart des Marxismus“20. In Thailand und Kambodscha liegen die Verhältnisse noch klarer; es hat z. B. in Thailand „kaum je marxistische Bewegungen von Bedeutung gegeben “21. In Laos scheint dagegen die Lage anders: hier gibt es in der Pathet-Lao eine starke kommunistisch beeinflußte Bewegung, die ihren ersten Programmpunkt „Schutz des Buddhismus als nationale Religion"22 teils nur dazu benutzt, die buddhistische Bevölkerung für sich zu gewinnen. Andererseits sind in dieser Partei aber auch starke nationale Kräfte vertreten, die wiederum den Marxismus benutzen, um sich gegen die amerikafreundlichen Politiker durchzusetzen und eine neutrale Politik des Königs zu ermöglichen. Wie stark die Rücksicht aller Parteien auf die buddhistische

\footnotetext{
16 Bechert II, S. 168.

17 E. Sarkisyanz, Südostasien seit 1945, München 1961, S. 92.

18 Bechert II, S. 135.

19 Bechert II, S. 160/161.

20 Bechert II, S. 161.

21 Bechert II, S. 202.

22 Bechert II, S. 295.
} 
Tradition ist, geht daraus hervor, daß Mönche die einzige Bevölkerungsgruppe sind, die in dem gespaltenem Land unbehelligt reisen können ${ }^{23}$.

6. Wenn auch die Lage in Laos in ihrer Verworrenheit sehr schwer zu durchschauen ist, so ist doch eines sicher, nämlich, daß die amerikanische Einmischung dem Lande nicht gedient hat. Wenn der amerikanische Botschafter in Laos sich rühmte, er habe 1956/57 sechzehn Monate lang dafür gekämpft, eine Regierungskoalition mit den Linksgruppen $\mathrm{zu}$ verhindern ${ }^{24}$, so ist diese Haltung nur aus folgenden Gründen verständlich: nach westlicher Auffassung ist jede Koalition mit den Kommunisten nur eine Vorbereitungshandlung, um die marxistische Alleinherrschaft zu errichten, wobei man das Beispiel der CSSR auch unausgesprochen im Sinne hat. Dabei sei nur am Rande erwähnt, daß es früher in anderen Staaten Koalitionen mit Kommunisten gegeben hat, z. B. die Volksfront in Frankreich, die keineswegs zu einer kommunistischen Diktatur führten. Vor allem aber wird bei einer solchen Betrachtungsweise der buddhistischen Tradition nicht genügend Rechnung getragen. Der Buddhismus kann dem Marxismus mit wissenschaftlichen psychologischen Argumenten entgegentreten, die in westlichen Weltanschauungen deshalb nicht oder nicht so stark vorhanden sind, weil diese ebenfalls auf materialistischen Prämissen aufbauen. Für den Buddhisten sind der Materialismus des Westens und sein Ableger, der Materialismus der Kommunisten, gar keine echten Alternativen, sondern zwei sich gegenseitig Zubringerdienste leistende Erscheinungsformen derselben Einseitigkeit. Der Buddhist ist überzeugt, die Wahrheit auf seiner Seite zu haben und sieht daher den Materialismus nur als vorübergehende Einseitigkeit an, wobei nach asiatisch-religiöser Auffassung mit viel größeren Zeiträumen gerechnet wird als bei uns. Eine politische Koalition mit Marxisten ist vom buddhistischen Blickpunkt aus in Laos ebenfalls nur eine Zwischenlösung, nämlich um die Kommunisten schließlich zu überzeugen und zurückzugewinnen für die buddhistische Gemeinsamkeit, die in der Weltpolitik „Neutralismus“ genannt würde.

7. Wie sehr Konflikte zwischen den Westmächten und dem Buddhismus aus Unverständnis herrühren, geht aus manchen Beispielen hervor. Nach buddhistischer Einsicht ist übermäßiges Erwerbsstreben als Habsucht verwerflich, weil dies von der eigentlichen menschlichen Aufgabe der Selbsterziehung ablenkt. Geld war z. B. außerhalb der Städte von Laos bis in die neueste Zeit völlig unbekannt ${ }^{25}$. Ein westlicher Beobachter, Norman Lewis, schrieb 1951 über Laos:

"The accumulation of wealth which is not to be used for definite, approved purposes, causes a man to lose prestige among neighbours, just, as in the West, the process is reversed."26

Von dort her kommen dann Urteile, welche die buddhistische Bevölkerung als faul und gemeinschaftsfeindlich kassifizieren und ihr mit Gewalt stattdessen die westlichen Errungenschaften des Konkurrenzkampfes usw. aufdrängen wollen. Als typisch kann hier die Schrift eines britischen Kolonialbeamten über Burma gelten: „Die wichtigste und größte Wahrheit heißt, den bestmöglichen Gebrauch von der schönen Welt, die Gott uns gegeben hat, zu machen. Die größte Sünde ist, die Erde zu belasten. Es ist unsere Pflicht, die Feigen, Untüchtigen, Schwachen, die die Erde mißbrauchen, fortzufegen und die Starken und Nützlichen an ihre Stelle zu setzen ... Der Burmese hat schon zu viel Glauben. Er ist zu viel gepflegt und

23 Bechert II, S. 295.

24 Bechert II, S. 277.

25 Bechert II, S. 265.

26 Zitiert nach Bechert II, S. 265. 
gehätschelt worden, man hat ihm $\mathrm{zu}$ viel gepredigt. Er muß aufstehen und kämpfen. Er soll nicht schaudern vor den Schlägen des Lebens und sich davor zurückziehen, sondern ... ihm entgegentreten. Er muß sein Wickelband des Glaubens abwerfen und den natürlichen Kämpfer in sich finden. Er muß lernen, wild zu sein, zu zerstören, wenn es nötig ist, ohne Skrupel zu verletzen, wegzustoßen. Er muß lernen, ein Mann zu sein. Ich kann mir nichts vorstellen, was den Burmesen so gut täte, als eins ihrer Regimenter sich in unseren Kriegen auszeichnen $\mathrm{zu}$ lassen. Es würde ihren Augen neue Aspekte des Lebens öffnen." ${ }^{27}$

Bei solchen Betrachtungen wird vergessen, daß die persönliche Genügsamkeit gerade dazu führt, nun um so mehr für die Gemeinschaft sozial wirken zu können. Die Unterstützung Notleidender und Alter erfolgt in den buddhistischen Ländern durch private Hilfe, ebenso die gesamte Unterhaltung des Ordens. In der alten Zeit verdankten in Burma nahezu alle Verkehrseinrichtungen ihr Dasein privater Wohltätigkeit ${ }^{28}$. Welchen Einfluß und welche Bedeutung diese caritative Seite des Buddhismus hat, läßt sich mit westlichen Maßstäben schwer erfassen, ist aber zum Verständnis der buddhistischen Staatslehre unerläßlich, die nur dann richtig $\mathrm{zu}$ würdigen ist, wenn die philosophischen Voraussetzungen der buddhistischen Weltanschauung deutlicher hervortreten ${ }^{29}$.

Hellmuth Hecker

27 Fielding Hall, A people at School, London 1906, S. 250 ff., zitiert nach Sarkisyanz, a. a. O., S. 87. 28 Bechert II, S. 13.

29 Das Werk von J. Schecter, The new face of Buddha. Buddhism and politcal power in Southeast Asia, London 1967, 298 S., konnte für den vorliegenden Aufsatz noch nicht berücksichtigt werden. 